\title{
Civilisations
}

Revue internationale d'anthropologie et de sciences

humaines

44 | 1997

Les peuples des forêts tropicales

\section{La nature des uns et la nature des autres}

Mythe et réalité du monde rural face aux aires protégées d'afrique centrale

\section{Daou V. Joiris}

\section{OpenEdition}

\section{Journals}

Édition électronique

URL : http://journals.openedition.org/civilisations/1613

DOI : 10.4000/civilisations. 1613

ISSN : 2032-0442

\section{Éditeur}

Institut de sociologie de l'Université Libre de Bruxelles

\section{Édition imprimée}

Date de publication : 1 janvier 1997

Pagination : 94-103

ISBN : 2-87263-122-4

ISSN : 0009-8140

Référence électronique

Daou V. Joiris, "La nature des uns et la nature des autres », Civilisations [En ligne], 44 | 1997, mis en ligne le 29 juin 2009, consulté le 01 mai 2019. URL : http://journals.openedition.org/civilisations/1613 ; DOI : 10.4000/civilisations. 1613 
-

\section{LA NATURE DES UNS}

ET LA NATURE DES AUTRES :

MUTHE ET RÉALITÉ OU MONDE RURAL

FACE AUX AIIRES PROTÉGEEES

D'AFFIOUUE CENTRALE

Da0u V. JOBRIS 


\section{Introduction}

Les nombreux témoignages relatifs aux relations tendues et parfois conflictuelles entre paysans et gestionnaires d'aires protégées ne peuvent laisser indifférents à une époque où la conservation manifeste une volonté croissante d'associer les populations locales à la gestion des zones préservées. En effet, à la suite de la mise sur pied de divers programmes de «gestion participative» tournant résolument le dos à une tradition écologiste d'expulsion des villages [particulièrement en Afrique de l'Est, voir Anderson \& Grove, 1987; Grove, 1987], on aurait pu s'attendre à des résultats encourageants. Or, c'est souvent avec un certain malaise que ces projets se résignent au constat sinon d'un échec, tout au moins d'une lourdeur dans les relations avec les riverains. Par ailleurs, force est de constater que même les programmes qui font état de certaines réussites ne résistent pas à une confrontation à la réalité, leurs expériences reposant malheureusement encore trop souvent sur un partenariat embryonnaire et fragile. Cet embarras résulte à notre sens d'une irréductible divergence d'intérêts entre gestionnaires et habitants des aires protégées, les objectifs des premiers étant en quelque sorte inconciliables avec les réalités et les aspirations des seconds. L'exemple que fournissent les difficultés avec lesquelles les décideurs tentent d'associer les populations locales d'Afrique Centrale à la gestion d'aires protégées telles que les Réserves de Faune du Dja, au Cameroun, la Réserve de la Lopé, au Gabon, et le Parc National d'Odzala en République du Congo (voir carte ci-dessous), se prête bien à l'analyse du cli-

Localisation de la Réserve de Faune du Dja, de la Réservce de Faune de la Lopé et du Parc National d'Odzala (Cameroun, Gabon, Congo).

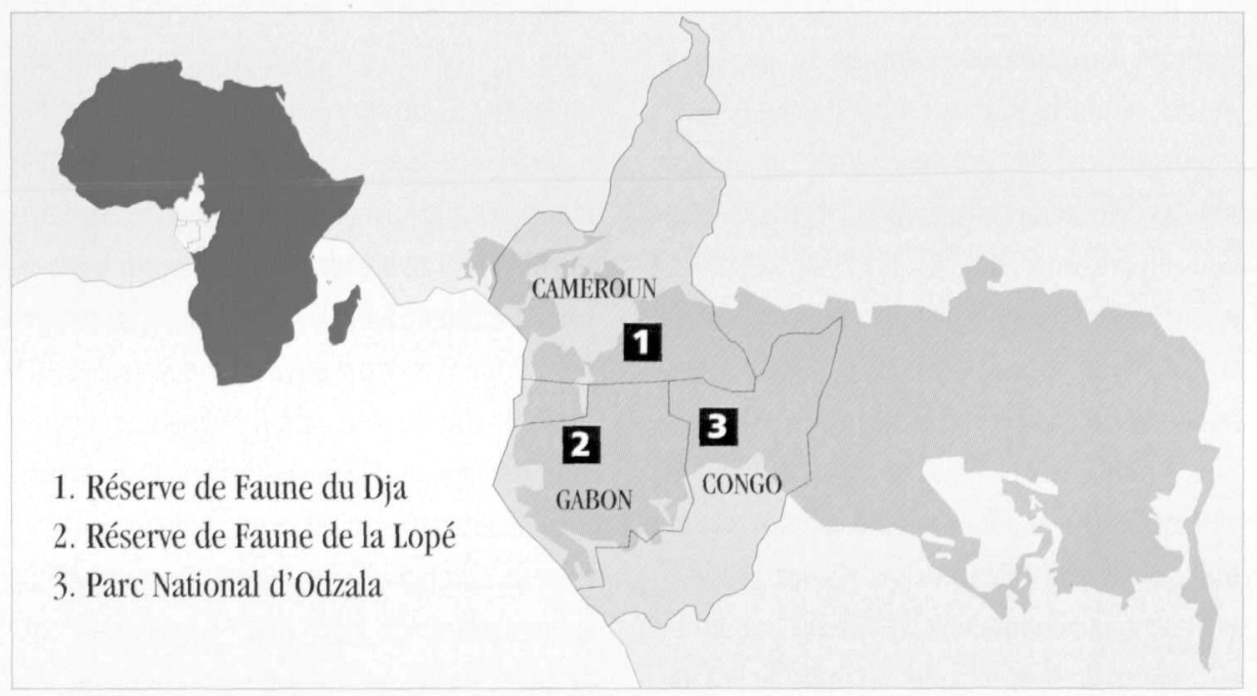


vage mis en l'œuvre dans un tel cadre. Il apparaît ainsi qu'en tant que champ d'application des législations occidentales, ces zones préservées sont souvent le thêâtre de l'incompréhension entre les tenants de deux registres de représentation et d'utilisation du milieu forestier; les décideurs ayant assigné une vocation touristique et scientifique à des terres qui jusque-là constituaient, et constituent encore, une source de vie pour ses habitants. Mais, en dehors de cette impasse, et comme en toile de fond de ce contexte, il nous semble que les compromis que pourraient octroyer les décideurs aux populations locales dans une tentative de réel partenariat sont rarement envisagés en raison d'un manque de dialogue entre " naturalistes" et paysans. Les éléments de réflexion que nous proposons ici ne se veulent ni exhaustifs, ni surtout moralisateurs, mais plutôt une tentative d'éclairage des réalités et représentations différentes en présence.

\section{Les protagonistes el les enjeux}

Dans le cadre des aires protégées d'Afrique Centrale, deux groupes constitués l'un par le personnel gérant la zone et l'autre par les paysans exploitant cette demière, représentent en quelque sorte les deux parties en présence. la première, que nous qualifierons ici de «naturaliste» et qui est généralement financée par des fonds occidentaux en collaboration avec des $\mathrm{ONG}$ et des ministères nationaux, regroupe non seulement des Occidentaux, mais aussi les Africains qualifiés de "Blancs », leur statut de salarié opérant comme un facteur de distinction vis-à-vis des riverains. Beaucoup plus nombreux, ces derniers, quant à eux, vivent de la terre et des produits de la forêt, grâce à des activités d'essartage, de culture de rente, de chasse, de pêche et de cueillettel. Du point de vue culturel, et pour les aires protégées qui nous concernent ici, il s' agit d'une mosaïque d'ethnies comportant d'un côté des chasseurs-cueilleurs en voie de sédentarisation (Pygmées Baka, Babongo et Bakola) et des essarteurs plus ou moins traditionnels encore plus diversifiés (Badjoué, Bulu, Simba, Mbahouin, Akele, Pouvi, Massango, Mboko, Mongom, Bakota, etc.) auxquels s'ajoutent une minorité de migrants spécialisés dans des activités commerciales (chasse, orpaillage, cultures de rente).

Mais d'une façon générale, et quelles que soient leurs spécificités culturelles, ces ethnies partagent globalement les mêmes conditions de vie en tant que sociétés paysannes dont l'économie dépend étroitement du milieu forestier; elles participent aussi de la même conception de la Nature. En ce qui les concerne, la forêt est vécue comme un milieu inhospitalier contre lequel il faut déployer une énergie et des savoir-faire considérables pour en exploiter les ressources et qu'il a fallu “domestiquer» (techniquement, symboliquement). Cette conception est diamétralement opposée à celle des «naturalistes» pour lesquels la même forêt, les mêmes espaces, ne représentent pas directement le cadre de vie ni ce qui leur permet de satisfaire leurs besoins; la forêt correspondant pour eux à un espace de découverte. Un exemple simple peut éclairer cet antagonisme: pour les paysans, l'éléphant évoque de la nourriture, de l'argent (ivoire) et un prédateur qui saccage les plantations sans parler des frayeurs que sa rencontre en forêt peut occasionner aux familles; pour les “ naturalistes », le même élé- 
phant renvoie principalement à un intérêt scientifique (espèce protégée) et financier (devises provenant du tourisme cynégétique et de vision), jamais à une menace pour leur sécurité ni à un produit alimentaire. Ceci est valable pour la plupart des animaux sauvages, dès lors qu'ils représentent un intérêt pour les «naturalistes». Nous sommes donc ici en présence de deux vécus qui conduisent inévitablement à des conceptions différentes de la Nature.

\section{La Nature des uns et la Nature des autres}

D'une manière générale, l'idée occidentale de la Nature mise en l'œuvre dans les pays du Sud s'inscrit à la fois dans la tradition républicaine et humaniste [Ferry, 1993] et dans les préoccupations de la « deep ecology » pour laquelle la question de la Nature prévaut sur celle de l'Homme [Host, 1994] ${ }^{\text {? }}$. Cette conception impose

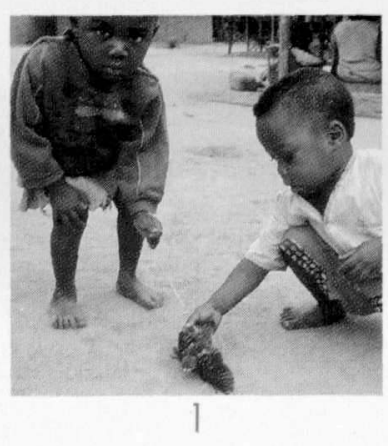

plusieurs jours de captivité pendant lesquels aucun soin ne lui est dispensé; les petits animaux et les oiseaux constituent aussi un jouet de prédilection pour les enfants (photo 1.) sans que personne ne s'émeuve de la souffrance de l'animal et une attitude amicale envers des bêtes est extrêmement rare, même vis-à-vis du chien de chasse. Par ailleurs, si l'on se situe au niveau social, l'idée indigène de la Nature s'inscrit dans un système relationnel (particulièrement à travers les relations de parenté entre vivants, et entre vivants et défunts) et non dans un système de rentabilité et d'exploitation de type occidental: souvent, une valeur particulière de prestige est attribuée aux animaux domestiques et une valeur symbolique au gibier.

Ce dernier, et les diverses modalités de son exploitation, constituent donc autant d'éléments qui mettent au jour une conception différente selon que l'on se place du point de vue du « naturaliste» ou du riverain. le respect des animaux sauvages, particulièrement les grands mammifères et la faune la plus exotique, et considère que les bêtes ont une sensibilité, des besoins et des droits. Par ailleurs, elle investit la Nature d'une valeur économique en l'intégrant dans le système occidental de rentabilité (exploitation industrielle; activités touristiques et cynégétiques) et en considérant son utilité potentielle en tant que laboratoire de la biodiversité (activités scientifiques).

Dans les villages, les populations locales n'ont pas la même sensibilité à l'égard des animaux. Il arrive que le gibier ne soit tué qu'après
La représentation que s'en fait celui-ci est considérée comme imparfaite par le premier qui, en dernier ressort, détient le pouvoir d'édicter et de faire respecter les règles du jeu. Ces conceptions divergeantes apparaissent particulièrement à travers un système d'interdits alimentaires et de représentation des animaux sauvages (valeurs et comportements) que les deux parties ont déve-

En général, les représentants de la conception occidentale s'abstiennent de consommer du gibier, sans pour autant adhérer à la rigueur du végétarisme et, au contraire, encouragent l'utililoppé suivant des logiques différentes. 
sation de la viande domestique. Ils disent ne pas s'adonner à la «viande de brousse » en raison de l'intérêt scientifique porté aux animaux sauvage, l'éthologie étant perçue comme incompatible avec la chasse. De plus, les programmes de conservation visent souvent à modifier le régime alimentaire des autochtones et à remplacer le gibier par le poisson et la viande domestique. Ainsi, les naturalistes considèrent que l'adoption du régime alimentaire local serait politiquement en contradiction avec leurs objectifs et, dans ce sens, leur position peut être qualifiée de militante. En présence d'Occidentaux et par souci d'intégration, les «naturalistes» ainsi que les cadres africains se plient au même comportement alimentaire. Quant au personnel local, des réserves de boîtes de conserve, de pâtes et de riz lui sont destinées pour ses sorties en forêt, ce qui a pour effet de l'encourager à ne plus consommer la viande sauvage.

\section{Dans la pratique des popu-}

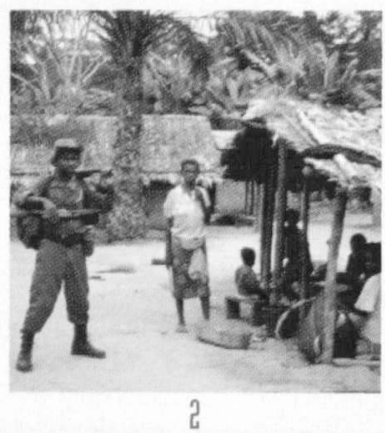

"Nous sommes nés dans la viande». L'interdiction de consommer tel ou tel animal procède d'un système très répandu dans les sociétés traditionnelles et qui fait « de la nature personnifiée en émotions, animaux et états psychologiques un acteur fondamental » [Da Matta, 1993 : 113]. Suivant cette interprétation, l'interdit alimentaire procède très souvent d'une logique métaphorique ou métonymique relative à certaines caractéristiques de l'animal [Levi-Strauss, 1962; de Garine, 1991; 1994].

Ainsi, l'interdit alimentaire portant sur le gibier s'inscrit dans un système de représentation différent suivant qu'il s'agisse du point de vue des populations locales. Pour les uns, l'interdit touche toutes les espèces et relève d'une humanisation de l'animal, tandis que pour les autres il ne touche que certaines espèces et il s'enracine dans un système particulier de représentation du monde. lations rurales, l'interdit alimentaire portant sur le gibier est d'un autre ordre. Il est sélectif dans le sens où il porte sur un nombre limité d'animaux dans des conditions variables: les populations distinguent les interdits temporaires auxquels l'individu se soumet pendant une période limitée (grossesse, initiation) et des interdits permanents que l'individu respecte pendant toute son existence. Mais ces prescriptions n'entrent pas en contradiction avec le fait que le gibier occupe une place importante dans l'alimentation locale; les habitants de ces zones forestières en mangent beaucoup car, comme ils le disent souvent: -

Quant aux animaux domestiques, en l'occurrence ceux que l'on rencontre dans les villages d'Afrique Centrale, c'est-à-dire principalement les ovins, les caprins et les volailles, ils ne font l'objet d'aucun soin particulier. Leur consommation est irrégulière, exceptionnelle. Il s'agit essentiellement d'un bien de prestige destiné aux échanges lors de prestations sociales telles les compensations matrimoniales et rituelles [voir Bahuchet, ce volume]. On tue une poule ou un mouton en l'honneur d'un visiteur de marque; on offre les mêmes animaux vivants à un parent à qui l'on doit le respect ou lors d'une cérémonie. Dans ce 
contexte, la valeur de prestige attribuée aux animaux domestiques differe de la conception occidentale suivant laquelle il s'agit d'une valeur strictement économique, alimentaire, voire affective. Lidée de la nature s'inscrit clairement ici dans la logique de systèmes sociaux où prévaut la dynamique relationnelle : la valeur du petit bétail et de la volaille dépassant celui d'un simple bien de consommation.

Les mêmes logiques divergentes s'inscrivent dans la relation entre la faune et l'Homme suivant que ce dernier est un riverain ou un "Blanc». Selon la taxonomie locale, la faune se répartit en trois catégories: les animaux bons à manger (le gibier, la «viande»), les animaux impropres à la consommation et les animaux "bons à penser" chargés d'une valeur symbolique, sur lesquels portent souvent des interdits alimentaires, mais qui ne sont pas nécessairement impropres à la consommation. Les catégories ethno-zoologiques varient d'une société à l'autre. À cette conception s'oppose celle des gestionnaires des aires protégées qui considèrent une autre partition de l'univers faunistique avec: les espèces protégées, les espèces non protégées et les «nuisibles". En général, les catégories locales et occidentales ne correspondent pas mais c'est la partition occidentale, inscrite dans la législation forestière, qui est imposée aux populations locales. Ainsi, beaucoup d'espèces protégées (taxonomie occidentale) correspondent à des animaux normalement consommés par les paysans alors que la plupart des espèces non protégées concernent du petit gibier relativement peu prisé dans l'alimentation locale [pour plus de détails sur l'alimentation en milieu forestier, voir Hladik \& al. 1989; 1993; Joiris, sous presse].
Venons-en maintenant à l'attitude vis-à-vis des animaux. De par son comportement non-prédateur, le "naturaliste" aborde la faune sous un autre angle que celui du chasseur autochtone et est ainsi perçu différemment par les animaux qui, dans les zones où ils ne sont pas chassés, à proximité des points d'eau, dans les salines, ne le craignent pas et sont encore moins agressifs. Le rapport à ces mêmes animaux est d'un autre ordre pour le chasseur autochtone qui utilise ses connaissances naturalistes pour piéger l'animal, le tuer, et qui, forcément, n'est jamais en présence que d'animaux sur la défensive. le cas du gorille est exemplaire: depuis que leur "apprivoisement » en milieu naturel s'est avéré un succès, les gestionnaires des aires protégées, dans leurs discussions avec les villageois sur la protection de l'espèce, tentent de convaincre ces derniers que l'animal est pacifique et, en dehors de cas de "légitime défense » ne prennent pas au sérieux l'insécurité dont les paysans se plaignent. Il en va de même pour d'autres espèces protégées, comme certains petits singes ou l'éléphant, qui n’entrent pas dans la catégorie des «nuisibles" mais qui détruisent pourtant les plantations [voir notamment Lahm, 1994]. C'est là un thème sensible dans les négociations entre les deux parties pour lequel les solutions proposées par les gestionnaires rencontrent rarement les préoccupations quotidiennes des plus directement concernés.

Ces exemples relatifs aux interdits alimentaires, à la valeur et aux comportements attribués aux animaux sauvages tantôt par les "naturalistes » et tantôt par les riverains, illustrent la nature d'un dialogue de sourd. Le malentendu, à la base, provient à notre sens d'une mauvaise 
connaissance de la culture des autochtones. Il n'est pas rare de constater que des programmes de gestion participante disposent de peu d'informations sur les ethnies en présence et que certaines généralisations conceptuelles sont imprégnées d' une représentation arbitraire des sociétés forestières. En général, le discours véhiculé par les programmes environnementaux en Afrique Centrale est double : la première tendance part de l'à priori que l'économie des populations locales est responsable de la destruction de la nature et qu'il faut dès lors les écarter des aires protégées, l'agriculture sur brûlis ainsi que la chasse, assimilée à du braconnage, représentant les principaux problèmes; la seconde approche, plus rare, postule exactement l'inverse, à savoir que leur économie contribue au maintien de la biodiversité et que l'on peut dès lors la maintenir sans risque dans les aires protégées. Mais quelle que soit l'orientation de ce double discours, il semble bien qu'il existe un clivage entre la rhétorique et la pratique et que, sur le terrain, tout se passe comme si la première tendance était d'application, les autorités compétentes traitant souvent de facto les populations comme si elles étaient réellement destructrices du milieu.

\section{Le "bon" et le "mauvais Sauvage"}

l'opinion occidentale classe les populations locales en «bon Sauvage» d'une part, en «maulvais Sauvage » d'autre part. Cette classification caricaturale apparaît dans les programmes de conservation pour lesquels le «bon Sauvage» est incamé par l'idée qu'on se fait du Pygmée vivant en harmonie avec son milieu [voir notamment la position de l'UICN, In Hecketsweiler \& al., 1991: 199], tandis que le «mauvais Sauvage» est assimilé à l'agriculteur sur brûlis (essarteur traditionnel) dont l'économie agricole, avec ses défrichements et ses brûlis, serait seule responsable de la destruction des forêts équatoriales.

Curiosité mythifiée en Occident depuis l'Antiquité avec le fameux texte d'Hérodote [voir Bahuchet, 1993], le Pygmée est aujourd'hui porteur de l'utopie rousseauiste selon laquelle il ferait "partie intégrante de l'écosystème forestier naturel » [Hecketsweiler \& al., op.cit.]. De cette assertion ressort qu'il est l'être humain le plus proche du monde animal, sa présumée économie archaïque sans machinisme l'apparentant considérablement à ce dernier. De-là à ce que le Pygmée soit donné à voir au même titre que les animaux exotiques d'un parc national ou d'une réserve naturelle, il n'y a qu'un pas que les tenants de l'écotourisme franchissent sans scrupule. Ainsi, une brochure touristique vantant les curiosités du Sud-Cameroun n'hésite pas à écrire que la zone présente un intérêt pour ses gorilles et ses ... Pygmées. De même, plus récemment, des organismes écologiques de renommée internationale présentent, sur le même plan, pour une affiche publicitaire, le portrait d'une femme Pygmée et les reproductions d'espèces protégées. Cette idée du Pygmée est bien évidemment le fait d'une représentation courante dont ces organismes ne sont que l'expression et dont il ne s'agit pas ici de faire le procès. Il n'en reste pas moins que de par son assimilation au «bon Sauvage», le Pygmée est présenté comme faisant partie d'une société, certes humaine, mais entièrement à l'intérieur du monde naturel. Faut-il encore démontrer qu' une telle représentation est fausse? Ces sociétés sont 
insérées, depuis des siècles, dans de vastes réseaux d'échange et sont donc loin d'appartenir à une civilisation préservée de tout contact [Bahuchet, ce volume]. Leur économie n'est plus celle de la civilisation du chasseur-cueilleur "authentique" et certaines techniques occidentales (fusils, tronçonneuses, insecticides) font maintenant partie de leur arsenal. La pêche à la nivrée, pour ne prendre que cet exemple, est actuellement facilitée par divers «poisons" industriels (provenant des exploitations forestières, insecticides du cacao) dont les usagers ignorent les effets toxiques. Enfin, la plupart des ethnies qu'elles représentent sont désormais fixées en bordure de piste et vivent dans des conditions qui s'apparentent progressivement à celles des essarteurs sur brûlis avec lesquels elles entretiennent d'ailleurs des relations socio-économiques depuis des générations.

Si les Pygmées sont tenus pour de grands connaisseurs et utilisateurs rationnels de la forêt, il n'en va pas de même pour les essarteurs traditionnels. Ces demiers sont pourtant peu connus dans la littérature ethnographique spécialisée, encore moins dans l'opinion publique et, de la même façon que pour ce qui vient d'être souligné pour les Pygmées, beaucoup d'idées les concernant sont erronées. D' après les monographies dont nous disposons, notamment sur les Mbo [Rösler, ce volume] et les Boyela [Sato, 1983 ] du Zaïre, ou sur les Mvae [Dounias, 1993] et les Badjoué [de Wachter, ce volume] du Cameroun, les essarteurs traditionnels d'Afrique Centrale ne pratiquent pas que l'agriculture itinérante sur brûlis, mais aussi la chasse, la collecte et la pêche. leur économie est complexe, elle nécessite des jachères très longues et l'entretien de vastes terroirs forestiers permettant d'assurer une exploitation pérenne et durable du milieu forestier dont on sait la grande fragilité. Certes, ces sociétés ont subi de lourds préjudices au cours de l'époque coloniale et post-coloniale ${ }^{[3]}$. Toutefois, on peut raisonnablement penser que si les écosystèmes forestiers tropicaux ont conservé jusqu'à ce jour leur étonnante diversité, c'est que, malgré tout, ces sociétés ont continué d'y exercer des économies adaptées. Ie discrédit dont l'agriculteur sur brûlis fait souvent l'objet relève d'une connaissance imparfaite de son économie. Souvent, l'opinion publique, relayée par celle des décideurs, cst informée des dégâts que cette technique agricole a occasionnés en Afrique de l'Ouest, par des cultures industrielles, ou en Amazonie, avec l'image de brûlis, provoquant ainsi une confusion entre l'agriculture itinérante et la culture intensive ou le défrichement permanent par le feu. Il est dès lors important de rappeler, ainsi que le montrent des approches agronomiques [de Wachter, op. cit.], et ethnoécologiques [Dounias, op.cit.] que les conditions d'une exploitation rationnelle semblent encore réunies dans la plupart des zones rurales d'Afrique Centrale ${ }^{[4]}$.

Mais la représentation négative de l'agriculteur sur brûlis a la vie dure. D'une certaine manière, elle conditionne les plans d'aménagement des ministères compétents. Ainsi, au Cameroun, le plan d'aménagement de la nouvelle légisJation forestière [Coté, 1992] n'octroie que $5 \mathrm{~km}$ de part et d'autre de la piste aux villages situés dans la «zone à exploitation ligneuse " (95\% de la superficie totale des forêts du Sud-Cameroun). Cette superficie est largement insuffisante: elle épouse systématiquement les routes suivant un alignement linéaire et ne prend pas en compte les 
jachères et les terroirs forestiers qui s'étendent audelà de la bande de $5 \mathrm{~km}$, au moins sur $20 \mathrm{~km}^{[3]}$. Un tel plan de zonage néglige totalement le caractère forestier de l'économie des essarteurs traditionnels, interdisant ainsi à l'avenir de pratiquer chasse, cueillette et pêche, activités pourtant indispensables, ne serait-ce qu'à une alimentation équilibrée et variée en milieu forestier. La situation des Pygmées, dont les aires d'exploitation sont plus vastes que celles des essarteurs, et dont la mobilité est plus forte, est encore plus dramatique. Dans le même ordre d'idée, certains ministères et organismes écologiques gabonais ont, par crainte des débordements de l'économie locale, appliqué, dans certaines aires protégées, un retrait des terroirs coutumiers qui a provoqué exactement l'effet inverse de ce que sont censés rechercher les environnementalistes. Ainsi, dans la Réserve de Faune de la Lopé, les institutions compétentes ont progressivement appliqué, depuis une quinzaine d'années, une politique interdisant les activités cynégétiques, y compris la pose des pièges dans les plantations. Le résuitat d'une telle politique est une forte augmentation de la prédation animale [Lahm, op.cit.] et une exploitation agricole non durable. Ies agricultrices ont en effet dû rapprocher les plantations de leurs villages afin de diminuer les risques de prédation et, pour celles qui le pouvaient, ont augmenté la superficie exploitée et réduit la durée des jachères. Aujourd'hui, suite à une "secondarisation" accélérée, les conditions suffisantes pour un épuisement progressif des sols en forêt dense et humide se trouvent vraisemblablement réunies.

La représentation négative de l'essarteur traditionnel conditionne aussi les politiques de déve- loppement rural. L'idée que l'agriculture sur brûlis est une technique agricole peu productive est un à priori très répandu. Les organismes de développement cherchent souvent à fixer les plantations et à en augmenter la production. Mais de telles mesures contribuent-elles vraiment à améliorer le système agricole?

Ces quelques exemples montrent, nous l'espérons, à quel point de simples représentations peuvent avoir une incidence sur les conditions de vie des populations locales. Il nous semble ainsi que l'articulation entre les catégories du «bon/mauvais Sauvage» et la supériorité de l'idée occidentale de la Nature, en tant qu'idéologie peu conforme à la réalité rurale, explique, tout au moins en partie, pourquoi les négociations avec les populations locales sont généralement mal ciblées. Souvent éludée, la question foncière est à nouveau un exemple parlant. Dans le cadre des aires protégées, le fait de ne considérer comme compatibles avec la gestion rationnelle des forêts que les économies Pygmées («bon Sauvage») conduit à n'envisager des plans d'aménagement, de développement, que pour ce type de population. Par-là, on tend à occulter l'existence d'autres groupes, en l'occurrence, les agriculteurs sur brûlis ( "mauvais Sauvage») qui correspondent pourtant à la majeure partie des ruraux [voir Introduction Régionale, ce volume]. Nier l'implantation de ces derniers équivaut évidemment à ne pas prendre en considération leur citoyenneté et, à fortiori, leurs droits d'usages coutumiers. Inutile de souligner qu'une politique de "gestion participative» qui repose sur de tels préceptes ne s'enracine pas dans des compromis acceptables pour les habitants des zones préservées. 


\section{Conclusion}

Le dêbat n'est pas de savoir s'il est bon ou non de protéger les forêts tropicales. Il s'agit d'initiatives pour lesquelles les nations et les populations locales portent une énorme responsathilité compte tenu de l'entreprise croissante du déboisement industriel et de la chasse commerciale. En réalité, l'enjeu est de savoir si, face à de telles contraintes, les habitants des aires protégées peuvent être réellement associés à la protection de leurs forêts. L'analyse de l'impact de programmes de conservation sur les populations locales ne peut faire l'économie, ni d'une approche politique globale, situant la problématique dans le cadre des relations Nord-Sud, ni d'un éclairage anthropologique, en s'interrogeant sur les registres de représentation et d'utilisation de la Nature en présence. Séparés les uns des autres par des conceptions sociologiques et culturelles différentes, les gestionnaires et les riverains des aires protégées ne partagent pas les mêmes objectifs dans un projet qui șe veut pourtant commun. Une meilleure connaissance et une meilleure écoute des réalités locales sont dès lors nécessaires pour établir des compromis acceptables pour les deux parties. Tel est certainement un des défis majeurs pour les décennies à venir.

\section{AEMERCIEMENTS}

Tous nos remerciements vont à nos collègues du progranme ECOFAC, alux personnes qui, dans la Réserve de Faune du Ija, dans la Réserve de Faune de la Loné et dans le Parc National d'Odzala nous ont accueillis et ont accepté de faire part de leurs vécus; ainsi qu à Serge [3ahuchet, Philippe Jespers et Paul Joiris pour les commentaires qu'ils ont bien voulu apporter à ce texte.

\section{LEEGENDES NES PHOTOS}

1 : Enfants du village de Mbanza jouant avec des oiseaux capturés (Parc National d'odzala, République du Congo).

? : Gardes forestiers devant une cuisine du village de Mbanza (Parc National d'odzala, Répuhlique du Congo).

\section{NOTES}

\section{1}

Présentées notamment, dans cee volume, dans les introductions générale [Bathuchet]), négionale [joiris] et dans des articies plus spécificques consacrés à l'agriculture itinérante sur brûlis [de Wachter] ou à l'économie d'une société d'essarteurs zairrois [Rösler].

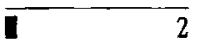

Il s'agit de la version nordaméricaine du mouvement de la "deep ecology" dont l'approche s'inscrit dans le prolongement de la perspective "préservationniste" selon laquelle la protection de la Nature est radicalement incompatible avec l'exploitation humaine

[Pimbert \& al., 1995: 15].

\section{- $-\frac{3}{3}$}

Tels que la désorganisation de la production locale pendant la période de traite. les réquisitions des honmes adultes pour des travaux forcés (routes, chemin de fer) et des activités militaires (guerres). la réduction de l'itinérance éd de la mobilité à la suite des rumembrements administratifs des villages cen bordure de piste, ainsi que l'introduction des nonocultures de rente provoquant un appauvrissement des sols.

\section{i- - 4}

Très faible densité de population, itinérance, exploitation de vastes superficies, système de rotation sur jachère de plus ou moins longue durée [Bahuchet; w: Wachter, ce volume].

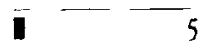

Notamment d'après nos estimations du terroir d'un village Badjoué dans la Réserve de Faune du Dja [Joiris \& Tchikangwa, 1995]. 